\title{
Bijections for Permutation Tableaux
}

\author{
Sylvie Corteel $^{1}$ and Philippe Nadeau ${ }^{2}$ \\ ${ }^{1}$ LRI,Université Paris-Sud, 91405 Orsay, France \\ ${ }^{2}$ Fakultät für Mathematik, Universität Wien, Nordbergstrasse 15, 1090 Vienna, Austria
}

\begin{abstract}
In this paper we propose a new bijection between permutation tableaux and permutations. This bijection shows how natural statistics on the tableaux are equidistributed to classical statistics on permutations: descents, RLminima and pattern enumerations. We then use the bijection, and a related encoding of tableaux by words, to prove results about the enumeration of permutations with a fixed number of 31-2 patterns, and to define subclasses of permutation tableaux that are in bijection with set partitions. An extended version of this work is available in [6].

Résumé. Dans cet article nous donnons une bijection entre les tableaux de permutations et les permutations. Cette bijection montre comment des statistiques naturelles sur les tableaux sont équidistribuées avec des statistiques classiques sur les permutations: descentes, minima de droite à gauche et motifs. Cette bijection nous sert ensuite, à l'aide d'un certain codage des tableaux par des mots, à donner des résultats sur l'énumération de permutations avec un nombre fixé de motifs 31-2, et à déterminer certaines sous-classes de tableaux en bijection avec les partitions d'ensembles. Une version étendue de ce travail est disponible [6].
\end{abstract}

Keywords: enumeration, bijections, permutations, tableaux, permutation patterns

\section{Introduction}

Permutation tableaux are fairly new objects that come from the enumeration of the totally positive Grassmannian cells $[12,15]$. Surprisingly they are also connected to a statistical physics model called the Partially ASymmetric Exclusion Process [5, 8, 9]. As in [13], a permutation tableau $T$ is a shape (the Ferrers diagram of a partition into non negative parts) together with a filling of the cells with 0's and 1's such that the following properties hold:

1. Each column contains at least one 1.

2. There is no 0 which has a 1 above it in the same column and a 1 to its left in the same row.

An example of a permutation tableau is given in Figure 1. Different statistics on permutation tableaux were defined in $[9,13]$. We list a few here. The length of a tableau is the number of rows plus the number of columns of the tableau. A zero in a permutation tableau is restricted if there is a one above it in the same column. A row is unrestricted if it does not contain a restricted entry. A one is superfluous if it contains a one above itself in the same column.

We label the South-East border of the shape of the tableau from 1 to its length, going from top-right to bottom-left. On Figure 1, a permutation tableau of shape $(3,3,3,3,1)$ and length 8 is given. The rows 1 , 3 and 7 are unrestricted and the rows 2 and 4 are restricted. 


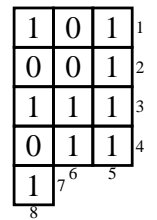

Fig. 1: Example of a permutation tableau

Our main interest here is that there exist $n$ ! permutation tableaux of length $n$. To our knowledge two bijections between permutations and permutation tableaux are known and appeared in $[2,13]$. The bijection given in [13] is quite complicated; but a lot of statistics of the permutation (weak excedances, crossings [5], alignments [15]... ) can be read from the tableau. In particular the set of weak excedances of the permutation corresponds to the set of rows of the tableau. See [13] for many more details. The bijection in [2] is the same as the one in [13], except that before applying the map some of the entries equal to one are changed into zero.

In this paper, we focus on descent statistics and generalized pattern enumeration and give a bijection between permutation tableaux and permutations.

Let us consider a permutation $\sigma=\left(\sigma_{1}, \ldots, \sigma_{n}\right)$ of $[n]=\{1,2, \ldots, n\}$. For $i<n$, we say that $\sigma_{i}$ is a descent if $\sigma_{i}>\sigma_{i+1}$, otherwise we call it an ascent. The shape of a permutation of $n$ is a partition $\lambda=\left(\lambda_{1}, \ldots, \lambda_{k}\right)$ with $\lambda_{k} \geq 0$ such that the $i^{t h}$ step of the boundary of $\lambda$ is West (resp. South) if $i$ is a descent (resp. ascent) of $\sigma$. For example, if $\sigma=(7,1,2,6,4,3,5)$, then the descents are 7,6 and 4 and the shape of $\sigma$ is $(3,3,3,2)$. As in [1], the generalized pattern $(31-2)$ occurs in $\sigma$ if there exist $i<j$ such that $\sigma_{i-1}>\sigma_{j}>\sigma_{i}$. The number of occurrences of $(31-2)$ is the cardinality of the set $\left\{1<i<j \mid \sigma_{i-1}>\sigma_{j}>\sigma_{i}\right\}$. In the previous example, $\sigma$ has six occurrences of the pattern $(31-2)$. An entry $\sigma_{i}$ is a $R L$-minimum of a permutation $\sigma$ if and only if $\sigma_{i}<\sigma_{\ell}$ for any $\ell>i$.

Our main result is the following:

Theorem 1 There exists a bijection $\xi$ between permutations of $[n]$ and permutation tableaux of length $n$. This bijection is such that if $T=\xi(\sigma)$ then

1. the shape of $T$ is the same as the shape of $\sigma$.

2. $i$ is an unrestricted row of $T$ if and only if $i$ is a RL-minimum of $\sigma$.

3. Thas superfluous ones if and only if there are $s$ occurrences of the pattern $(31-2)$ in $\sigma$.

Remark. Theorem 1 without item (2) is implied by the composition of the two bijections presented in [13]. Our map is different from this composition or any variation of it and gives the full Theorem 1.

In Section 2, we give a very simple enumerative result showing that there are $n$ ! permutation tableaux of length $n$. We present in Section 3 a first bijection between permutation tableau and permutations which gives Theorem 1 without item (3). To prove Theorem 1, we describe the bijection in Section 4. We give some applications to pattern enumeration in Section 5, define some families of tableaux counted by Bell numbers in Section 6 and we conclude in Section 7. 


\section{How many tableaux?}

Let $t(n, k, \ell)$ be the number of tableaux of length $n$ with $k+1$ unrestricted rows and $\ell$ ones in the first row, and let $T_{n}(x, y)=\sum_{k, \ell} t(n, k, \ell) x^{k} y^{\ell}$.

Proposition 1 If $n>1$,

$$
T_{n}(x, y)=\prod_{i=0}^{n-2}(x+y+i)
$$

and $T_{1}(x, y)=1$. In particular $T_{n}(1,1)$, the number of tableaux of length $n$, is equal to $n$ !.

The proof can be found in [6]: it uses a decomposition of tableaux according to their first column to get a recurrence for the numbers $t(n, k, \ell)$. This implies in particular $T_{n}(x, y)=T_{n}(y, x)$ and we get a symmetry result which was proved combinatorially in [9].

Corollary 1 The number of permutation tableaux of length $n$ with $k+1$ unrestricted rows and $\ell$ ones in the first row is equal to the number of tableaux of length $n$ with $\ell+1$ unrestricted rows and $k$ ones in the first row.

The proposition also implies a result proved in [7] thanks to the bijection of [13] :

Corollary 2 The number of permutation tableaux of length $n$ with $k+1$ unrestricted rows (or $k$ ones in the first row) is equal to the first Stirling number $s(n, k)$ which enumerates the number of permutations of $[n]$ with $k$ cycles.

\section{Bijection I}

In this section we exhibit a first bijection between permutation tableaux of length $n$ and permutations of $[n]$. This bijection is simple, and verifies the first two items of Theorem 1.

A zero in a permutation tableau is a rightmost restricted zero if it is a restricted zero and there is no restricted zero to its right in the same row. The bijection relies on the following simple claim: a permutation tableau is uniquely determined by its topmost ones and rightmost restricted zeros. Indeed if one knows the positions of the topmost ones (resp. rightmost restricted zeros), then all the cells above them (resp. to their left) are filled with zeros. The rest of the cells are filled with superfluous ones.

The bijection. We start with the tableau $T$ of shape $\lambda$. Then we initialize the permutation $\sigma$ to the list of the labels of the unrestricted rows in increasing order. Now for each column, starting from the left proceeding to the right, we perform the following: let $j$ be the label of the column, and $(i, j)$ its topmost 1. Then we insert $j$ to the left of $i$ in the permutation $\sigma$, and if the column contains rightmost restricted zeros in rows $i_{1}, \ldots, i_{k}$, we insert $i_{1}, \ldots, i_{k}$ in increasing order to the left of $j$ in the permutation $\sigma$.

Example. We start with the tableau in Figure 1. The unrestricted rows are rows 1,3 and 7. The rightmost restricted zeros are in cells $(2,8)$ and $(4,8)$. We start with the permutation $(1,3,7)$, We insert 8 to the left of 1 and insert 2 and 4 to the left of 8 . We get $(2,4,8,1,3,7)$. We insert 6 to the left of 3 and get $(2,4,8,1,6,3,7)$. Finally we insert 5 to the left of 1 . The permutation is $(2,4,8,5,1,6,3,7)$. 
The reverse is as easy to define, and is explicited in [6]. We have thus defined in this Section a simple bijection that possesses the first two properties of Theorem 1; to get all three properties, we will define another bijection in a quite different way.

\section{Main Bijection}

We will define notions of reduction for tableaux and permutations, and from these we will be able to build our second bijection

\subsection{Reduction of a tableau}

We give in this subsection a recursive decomposition of the tableaux that was used in [15] to enumerate permutation tableaux with two rows. This decomposition will be essential to define our second bijection.

Let $T$ be a tableau of length $n>0$ and of shape $\left(\lambda_{1}, \lambda_{2}, \ldots, \lambda_{m}\right)$. We suppose that the last row of $T$ is labeled by $k$ and that the length of this row is $t$. Then three cases are possible:

- Type 1 : The last row does not contain any ones.

- Type 2 : The rightmost entry of the last row contains a topmost one.

- Type 3 : The rightmost entry of the last row contains a superfluous one.

From the definition of the permutation tableaux we know that these are the only three possible cases. Indeed if the rightmost entry of the last row is a zero then all the entries of the row are zeros.

We can then reduce a tableau $T$ according to its type:

- If the tableau $T$ is of type 1 , then we can delete the last row and get a tableau of length $n-1$ and shape $\left(\lambda_{1}, \ldots, \lambda_{m-1}\right)$.

- If the tableau is of type 2 , then we can delete the column $k+1$ and get a tableau of length $n-1$ and shape $\left(\lambda_{1}-1, \ldots, \lambda_{m}-1\right)$.

- If the tableau is of type 3, we can delete the rightmost entry of the last row and get a tableau of length $n$ and shape $\left(\lambda_{1}, \ldots, \lambda_{m-1}, \lambda_{m}-1\right)$.

The resulting tableau is denoted $\operatorname{red}(T)$; note that when applying this reduction, the sum of the length of the tableau plus its number of superfluous ones decreases by one. Therefore, given a tableau of length $n$ with $j$ superfluous ones, exactly $n+j$ reductions will give the empty tableau. If each time we reduce the tableau, we keep in mind the type $1(t)$ ( $t$ is the length of the last row), 2 or 3 , this gives an encoding of the tableau, since it allows us to inverse the specific reduction that took place.

Let us give a simple example in Figure 2. The tableau of shape $(2,2,2)$ at the extreme right is reduced successively, and 1(0), 2, 2,1(2),1(0), 3,3 is the code obtained in the process.

\subsection{Reduction of a permutation}

Given a permutation $\sigma=\left(\sigma_{1}, \ldots, \sigma_{n}\right)$ with $\sigma_{j}=k$, we denote by $(31-2)(k)$ the cardinality of the set $\left\{1<i<j \mid \sigma_{i-1}>k>\sigma_{i}\right\}$. This corresponds to the number of occurrences of the pattern 31-2 where $k$ is the "2" of the pattern. For example, if $\sigma=(5,2,1,6,3,4)$ then $(31-2)(4)=2$. Let $\sigma$ be a permutation 


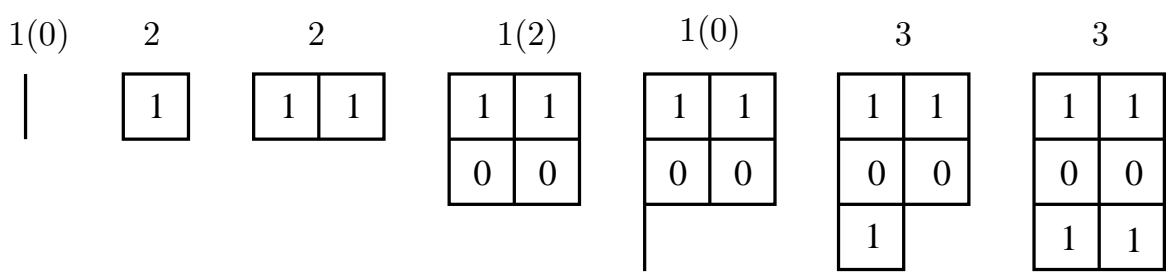

Fig. 2: Successive reductions of a tableau (from right to left).

of shape $\lambda=\left(\lambda_{1}, \ldots, \lambda_{m}\right)$ such that $k$ is the largest ascent. We suppose that $\sigma_{0}=0$ and $\sigma_{n+1}=n+1$. We say that $\sigma_{i}$ is a peak (resp. double descent, resp. valley, resp. double ascent) if $\sigma_{i-1}<\sigma_{i}>\sigma_{i+1}$ (resp. $\sigma_{i-1}>\sigma_{i}>\sigma_{i+1}$, resp. $\sigma_{i-1}>\sigma_{i}<\sigma_{i+1}$, resp. $\sigma_{i-1}<\sigma_{i}<\sigma_{i+1}$ ).

Three types of permutations exist :

- Type $1: k$ is a double ascent in $\sigma$ and $(31-2)(k)=0$.

- Type $2: k$ is to the right of $k+1$ in $\sigma$ and $(31-2)(k+1)=0$ and one of the following holds

$-k+1$ is a double descent

$-k$ and $k+1$ are adjacent

- Type 3 : None of the previous configurations appears. That is

1. $k$ is a valley and is adjacent to $k+1$ and to its left; or

2. $k+1$ is a peak and $k$ is just to the right of $k+1$ and $(31-2)(k+1)>0$; or

3. $k$ is to the left of $k+1$ and $k$ is a double ascent and $(31-2)(k)>0$; or

4. $k+1$ is to the left of $k$ and $k+1$ is a double descent and $(31-2)(k+1)>0$; or

5. $k$ is a valley and is to the left of $k+1$ but not adjacent to it; or

6. $k+1$ is a peak and is to the left of $k$ but not adjacent to it .

This takes care of all the possible cases.

We define a reduction $R E D$ of the permutation $\sigma$ whose largest ascent is $k$ :

- If $\sigma$ is of type 1 : Delete $k$ and decrease by one all the entries greater than $k$. The result is a permutation of $[n-1]$ and shape $\left(\lambda_{1}, \ldots, \lambda_{m-1}\right)$.

- If $\sigma$ is of type $2:$ delete $k+1$ and decrease by one all the entries greater than $k$ and get a permutation of $[n-1]$ and shape $\left(\lambda_{1}-1, \ldots, \lambda_{m}-1\right)$.

- If $\sigma$ is of type 3 : apply bijection $\Phi$ defined below and get a permutation of $[n]$ and shape $\left(\lambda_{1}, \ldots, \lambda_{m-1}, \lambda_{m}-\right.$ 1) with one less occurrence of $(31-2)$. 
The rest of this subsection is devoted to giving a bijection $\Phi$ between permutations of $[n]$ of type 3 of shape $\lambda$ with $j$ occurrences of $(31-2)$ and permutations of shape $\left(\lambda_{1}, \ldots, \lambda_{m-1}, \lambda_{m}-1\right)$ with $j-1$ occurrences of $(31-2)$. The basic idea is to exchange $k$ and $k+1$ in $\sigma$ in order to transform $k$ into a descent, $k+1$ into an ascent. This will work unless $k$ and $k+1$ are adjacent. Moreover we will decrease by one the number of occurrences of $(31-2)$, unless $k$ is to the left and not adjacent to $k+1$ or $k$ is adjacent to $k+1$ and to its right. In those cases, we will have to do a bit more.

We give the details in the following paragraph and illustrate in parallel the bijection on Figure 3 . We write the permutation $\sigma=\left(\sigma_{1}, \ldots, \sigma_{n}\right)$ as the word $0 \sigma_{1} \ldots \sigma_{n}(n+1)$. We suppose that $p_{1}, p_{2}, \ldots$ are words with elements smaller than $k ; G_{1}, G_{2}, \ldots$ are words with elements larger than $k$; and that $X, Y, Z$ are words. The words denoted $X, Y, Z$ may be empty, while the $p_{i}$ and $G_{i}$ are nonempty unless explicitly stated otherwise:

1. If $k$ is a valley and is adjacent to $k+1$ and to its left, then $\sigma$ can be written as $X p_{1} G_{1} k(k+1) p_{2} Y$. We set $\Phi(\sigma)=X p_{1}(k+1) G_{1} k p_{2} Y$.

2. If $k+1$ is immediately to the left of $k$ and is a peak and $(31-2)(k+1)>0$, then $\sigma$ can be written as $X G_{1} p_{1}(k+1) k G_{2} Y$. We set $\Phi(\sigma)=X G_{1} k p_{1}(k+1) G_{2} Y$.

3. If $k$ is to the left of $k+1$ and $k$ is a double ascent and $(31-2)(k)>0$, then $\sigma$ can be written as $X p_{1} G_{1} p_{2} k G_{2} Y(k+1) p_{3} Z$. We set $\Phi(\sigma)=X p_{1}(k+1) G_{1} p_{2} G_{2} Y k p_{3} Z$. (Here $G_{2} Y$ may be empty.)

4. If $k+1$ is to the left of $k$ and $k+1$ is a double descent and $(31-2)(k+1)>0$, then $\sigma=$ $X G_{1} p_{1} G_{2}(k+1) p_{2} Y k G_{3} Z$ and $\Phi(\sigma)=X G_{1} k p_{1} G_{2} p_{2} Y(k+1) G_{3} Z$. (Here $p_{2} Y$ may be empty.)

5. If $k$ is a valley and is not adjacent to $k+1$ and to its left, then $\sigma$ can be written as $X p_{1} G_{1} k G_{2} Y(k+$ 1) $Z$. We set $\Phi(\sigma)=X p_{1} G_{1}(k+1) G_{2} Y k Z$.

6. If $k+1$ is a peak and is not adjacent to $k$ and to its left, then $\sigma$ can be written as $X p_{1}(k+$ 1) $p_{2} Y k G_{3} Z$. We set $\Phi(\sigma)=X p_{1} k p_{2} Y(k+1) G_{3} Z$.

The six cases are pictured on Figure 3. The dots represent $k$ and $k+1$, and possible prefixes and suffixes are not pictured since they are not modified by $\Phi$.

Proposition $2 \Phi$ is a bijection between permutations of $[n]$ of type 3 of shape $\lambda$ with $j$ occurrences of $(31-2)$ and permutations of shape $\left(\lambda_{1}, \ldots, \lambda_{m-1}, \lambda_{m}-1\right)$ with $j-1$ occurrences of $(31-2)$

The proof of this can be found in [11]. From this result, we will be able to derive an algorithmic bijection between permutation tableaux and permutations. This is what we explain in the following section.

\subsection{The bijection $\xi$}

From permutations to tableaux. Let $\sigma$ be a permutation of $[n]$ and $k$ its largest ascent. If $\sigma$ is the empty permutation then $\xi(\sigma)$ is the empty tableau.

Otherwise we define $\xi(\sigma)$ by induction. Let $T^{\prime}$ be the tableau $\xi(R E D(\sigma))$.

- If $\sigma$ is of type $1: \xi(\sigma)$ is the tableau $T^{\prime}$ with one extra row of length $n-k$ filled with zeros. 


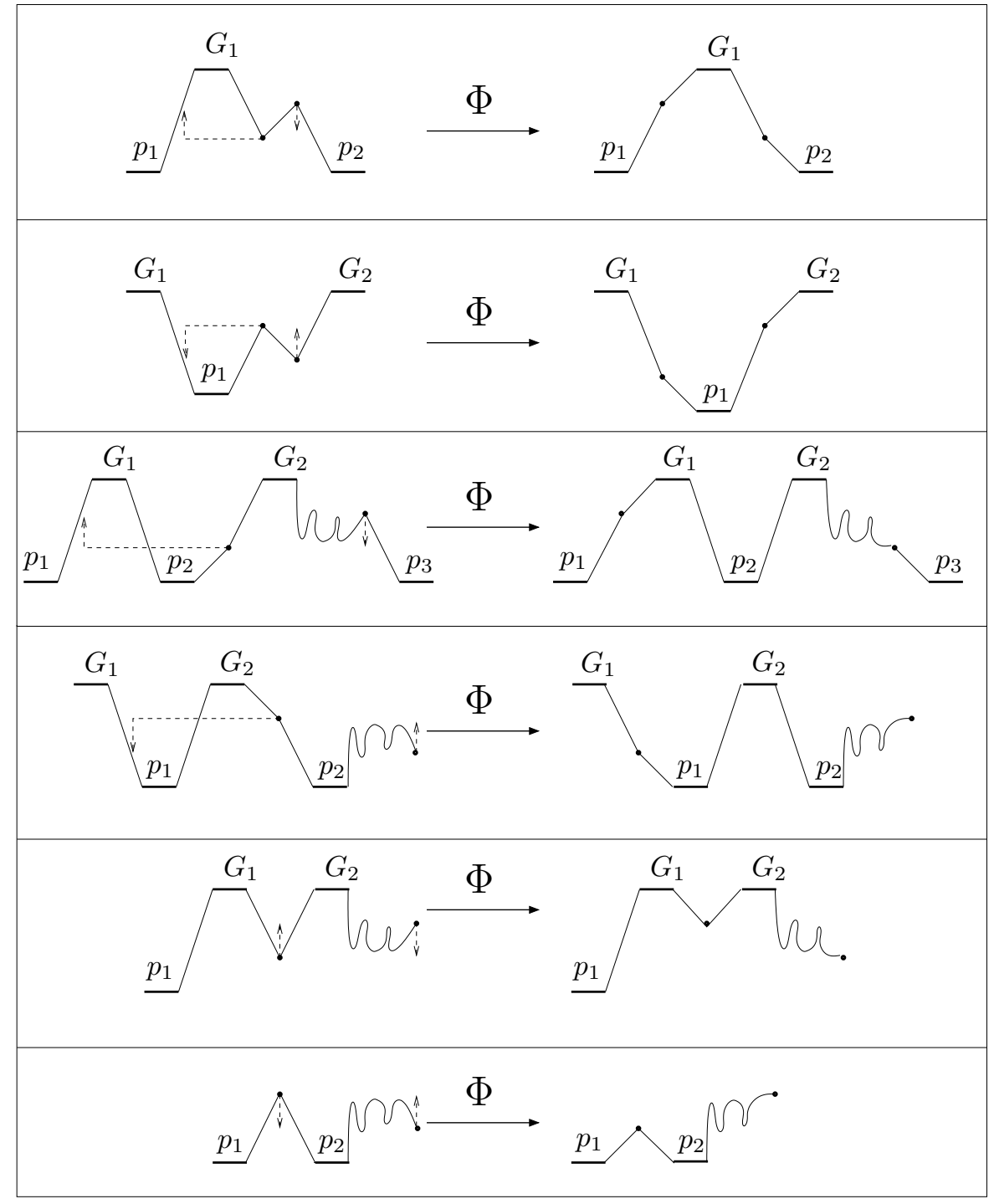

Fig. 3: The six cases in the definition of $\Phi$. 
- If $\sigma$ is of type 2: $\xi(\sigma)$ is the tableau $T^{\prime}$ with one extra column made of as many rows as $T^{\prime}$ with its lower cell at the end of the last row of $T^{\prime}$. This lower cell is filled with a one and all the cells above it with zeros.

- If $\sigma$ is of type $3: \xi(\sigma)$ is the tableau $T^{\prime}$ with one extra cell added to the last row and filled with a superfluous one.

This can be best expressed by the encoding described at the end of paragraph 4.1: if $c$ is the list encoding the tableau $T^{\prime}$, then when $\sigma$ is of type 1 (resp. of type 2, resp. of type 3), we define the encoding of $T$ by $T=c, 1(n-k)($ resp. $T=c, 2$, resp. $T=c, 3)$

Example. If we start with the permutation 25143, then we have the following successive reductions, where in each case we underline the corresponding entries $k$ and $k+1$ (if $k<n$ ) involved in the reduction, and we indicate the type of the permutation:

$251 \underline{43}$, type $3 ; 2 \underline{5} 31 \underline{4}$, type $3 ; 2431 \underline{5}$, type $1 ; \underline{2} 4 \underline{3} 1$, type $1 ; 3 \underline{21}$, type $2 ; \underline{21}$, type $2 ; \underline{1}$, type 1 .

Then by reconstructing the tableau, we obtain exactly the encoding shown on the top of Figure 2 , and the tableau $\xi(\sigma)$ is thus the tableau on the right of the Figure.

Proof of Theorem 1 (sketch). One first proves by induction (see [6]) that

1. the shape of $T=\xi(\sigma)$ is the same as the shape of $\sigma$.

2. $i$ is an unrestricted row of $T$ if and only if $i$ is a RL-minimum of $\sigma$.

3. $T$ has $s$ superfluous ones if and only if there are $s$ occurrences of the pattern $(31-2)$ in $\sigma$.

We then need to prove that $\xi$ is indeed a bijection; for this, we give the reverse mapping, where we will use the notations $p_{i}, G_{i}, X, Y$ introduced in the definition of the function $\Phi$.

From tableaux to permutations. If $T$ is the empty tableau then $\xi^{-1}(T)$ is the empty permutation. Otherwise we will define $\xi^{-1}(T)$ by induction; let $\sigma$ be the permutation $\xi^{-1}(\operatorname{red}(T))$ :

- If $T$ is of type 1 and its last row is of length $n-k:$ increase all the entries of $\sigma$ greater than or equal to $k$ by one. Insert $k$ to the left of the leftmost entry greater than $k$, so that we transform $p_{1} G_{1} X$ in $p_{1} k G_{1} X$.

- If $T$ is of type 2, then let $k$ be the largest ascent of the permutation $\sigma$. Increase by one all the entries greater then $k$.

1. If there is no entry larger than $k$ to its left, then insert $k+1$ to the left of $k$; that is, we transform $p_{1} k X$ in $p_{1}(k+1) k X$.

2. Otherwise let $i$ be the leftmost element greater than $k$ such that $i$ is to the left of $k$ and the element after $i$ is smaller than $k+1$. Insert $k+1$ to the right of $i$ in $\sigma$ : thus we transform $p_{1} G_{1} X k Y$ in $p_{1} G_{1}(k+1) X k Y$.

- If $T$ is of type 3 then $\sigma$ becomes $\Phi^{-1}(\sigma)$.

In each case the permutation $\xi^{-1}(T)$ is defined to be the permutation $\tau$ obtained; it is respectively of type 1,2 and 3, and $R E D(\tau)$ is exactly the permutation $\sigma$; see [11] for details. This proves Theorem 1 . 


\section{Permutation patterns}

\subsection{Bijection between permutation tableaux and PT-words}

We will show that the reduction defined in Section 4 directly defines a bijection $\Upsilon$ between permutation tableaux and certain words on the alphabet $\{D, U, V\}$. We define the height $h$ of the letters $h(D)=-1$, $h(U)=h(V)=1$. The height of a word is the sum of the heights of its letters. To define $\Upsilon$, it is easier to define first a function $\Upsilon_{0}$ as follows: if $T$ is the empty tableau then $\Upsilon_{0}(T)$ is the empty word. Otherwise, let $t$ be the length of the last row of $T$ :

- If $T$ is of type 1 , then $\Upsilon_{0}(T)=\Upsilon_{0}(\operatorname{red}(T)) D^{i} U$, where $i$ is such that $h\left(\Upsilon_{0}(T)\right)=i+1$.

- If $T$ is of type 2 , then $\Upsilon_{0}(T)=\Upsilon_{0}(\operatorname{red}(T)) U$.

- If $T$ is of type 3, then $\Upsilon_{0}(T)=\Upsilon_{0}(\operatorname{red}(T)) V$,

where $\operatorname{red}(T)$ is the reduction defined in Section 4.1 .

We append $t+1$ letters $D$ at the end of $\Upsilon_{0}(T)$ if the last row of $T$ has length $t$, and this gives us finally the word $\Upsilon(T)$.

Example 3. Consider the tableau $T_{0}$ on the extreme right of Figure 2, the word $\Upsilon_{0}\left(T_{0}\right)$ is $U \cdot U \cdot U \cdot D U$. $D D D U \cdot V \cdot V$, and one appends $D D D$ at the end to obtain the final word $\Upsilon(T)=U U U D U D D D U V V D D D$. To take a bigger example, consider the tableau $T_{1}$ of Figure 1 . We have $\Upsilon\left(T_{1}\right)=\Upsilon_{0}\left(T_{1}\right) D D$ because the last row of $T_{1}$ has length 1 . Then one checks that

$$
\Upsilon_{0}\left(T_{1}\right)=U U U D D U V D D D U V U V D D D U V V D D D D U V .
$$

We explicit the family of words given by this construction: a PT-word is a word $w$ on the alphabet $\{D, U, V\}$ such that:

- $h(w)=0$ and $h(X) \geq 0$ for each prefix of $w$;

- a letter $D$ can not be followed by a letter $V$;

- for each factor $D^{d+1} U M$ with $M$ a word on the alphabet $\{U, V\}$ and $d$ chosen maximal, $M$ contains at most $d$ letters $V$;

- only letters $U$ are allowed to precede the first letter $D$.

Proposition $3 \Upsilon$ is a bijection between permutation tableaux of length $n, k$ superfluous ones and $j$ unrestricted rows and PT-words of length $2 n+2 k$, with $k$ letters $V$ and $j$ prefixes of height 0 .

\subsection{Shape of a tableau $T$ given $\Upsilon(T)$}

We can easily describe the shape of a tableau $T$ given its associated PT-word $\Upsilon(T)$ : if $\Upsilon(T)$ is empty then $T$ is the empty tableau. Otherwise, decompose $\Upsilon(T)$ in the form

$$
\Upsilon(T)=U^{k_{0}} D^{l_{1}} M_{k_{1}} \cdots D^{l_{t}} M_{k_{t}} D^{l_{t+1}}
$$

where all $k_{i}$ and $l_{i}$ are positive, and $M_{k_{i}}$ is a word on the alphabet $\{U, V\}$ for each $i$. Define $v_{i}$ as the number of letters $V$ in the word $M_{k_{i}}$; by definition of a PT-word we have $v_{i} \leqslant l_{i}-1$. Then the South East border of the tableau $T$ is given by

$$
S W^{l_{1}-1-v_{1}} S W^{l_{2}-1-v_{2}} S \cdots W^{l_{t}-1-v_{t}} S W^{l_{t+1}-1} .
$$


This is easily proved by induction.

For the word $\Upsilon(T)$ of Example 3, we have $l_{1}=2, v_{1}=1 ; l_{2}=3, v_{2}=2 ; l_{3}=3, v_{3}=2 ; l_{4}=4, v_{4}=$ 1 and finally $l_{5}=2$. This gives a South East border encoded by $S S S S W W S W$, in concordance with the tableau of Figure 1.

\subsection{One occurrence of $(31-2)$}

It is well known that the number of permutations of $[n]$ with no occurrence of the pattern $(31-2)$ is equal to the $n^{\text {th }}$ Catalan number [4] . The bijection between permutation tableaux and PT-words given in Section 5.1 gives another proof of this fact. Indeed if the permutation tableau has no superfluous ones, the corresponding word is a Dyck word. Thanks to this approach, we can also give the first combinatorial proof of the following fact :

Proposition 4 [4] The number of permutations of $[n]$ with one occurrence of the pattern $(31-2)$ is equal to

$$
\left(\begin{array}{c}
2 n \\
n-3
\end{array}\right) \text {. }
$$

The bijective proof (see [6]) uses the standard techniques of cycle lemma and the André reflexion principle to go bijectively from PT-words of length $2 n+2$ with one letter $V$, to words on $\{D, U\}$ of length $2 n$ that end at height -6 . It would be interesting to pursue this approach to give combinatorial proofs of the following facts proved analytically by Claesson and Mansour.

Proposition 5 [4] The number of permutations of [ $n]$ with two (respectively three) occurrences of $(31-2)$ is

$$
\frac{n(n-3)}{2(n+4)}\left(\begin{array}{c}
2 n \\
n-3
\end{array}\right) \quad\left(\text { resp. } \frac{1}{3}\left(\begin{array}{c}
n+2 \\
2
\end{array}\right)\left(\begin{array}{c}
2 n \\
n-5
\end{array}\right)\right)
$$

\section{Bell tableaux}

In this Section we give two subfamilies of permutation tableaux that are in bijection with set partitions. A set partition of the set $[n]$ is a set of pairwise disjoint subsets of $[n]$ whose union is $[n]$. A set partition can also be seen as a permutation where all the cycles are increasing cycles. Recall that a one is topmost if it has no ones above itself in its column. A one is leftmost if it has no ones to its left in its row and rightmost if it has no ones to its right in its row.

Definition 1 An R-Bell tableau (respectively an L-Bell tableau) is a permutation tableau where all the topmost ones are also rightmost ones (resp. leftmost ones).

Proposition 6 There exists a bijection between L-Bell tableaux of length $n$ such that the sum of the number of columns and the number of zero rows is $k$ and set partitions of $[n]$ with $k$ blocks.

Proof: For every column of the tableau, construct a block of the set partition that is made of the label of the column and the labels of the rows that have a one in this column which is the leftmost one of its row. The reverse is as easy to define.

For example, given the tableau on Figure 4, we get the set partition $\{1,7,8\},\{3,4,6\},\{2,5\}$. 


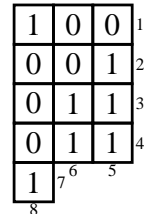

Fig. 4: Example of a tableau where the topmost ones are also leftmost

Proposition 7 There exists a bijection between $R$-Bell tableaux of length $n$ and $k$ rows and set partitions of $[n]$ with $k$ blocks.

Proof: We propose a bijection based on the bijection of [13]. We apply this bijection to construct a permutation $\sigma$. This bijection is such that for each row with label $i$, if the row has no ones then $\sigma(i)=i$. Otherwise start with the leftmost one of row $i$ and travel South and East changing direction each time a one is reached until the border is reached. Then $\sigma(i)=j$, where $j$ is the label of the border. Apply the same process for the columns, starting at the topmost one and traveling East and South. It is easy to see that the tableau is an R-Bell tableau if and only if $\sigma(i)<i$ implies that $\sigma(\sigma(i)) \geq \sigma(i)$ and there does not exist $j<i$ such that $\sigma(j)<\sigma(i)<j<i$. Then we can transform $\sigma$ in the set partition $\Pi=\left\{\Pi_{1}, \ldots, \Pi_{k}\right\}$ such that $k$ is the number of non excedances plus the number of fixed points of $\sigma$ and such that in each block $\left\{\pi_{1}, \pi_{2}, \ldots, \pi_{\ell}\right\}$ then $\left(\ell=1\right.$ and $\left.\sigma\left(\pi_{\ell}\right)=\pi_{\ell}\right)$ or $\pi_{i}=\sigma\left(\pi_{i-1}\right)$ for all $1 \leq i<\ell$ and $\sigma\left(\pi_{\ell}\right)<\pi_{\ell}$.

One might be surprised that R-Bell and L-Bell tableaux of length $n$ are both in bijection with set partitions of $[n]$ : though their definition looks symmetric, there is no apparent left-right symmetry in the definition of a permutation tableau. We can indeed show directly that

Proposition 8 There is a bijection between R-Bell tableaux of shape $\lambda$ and L-Bell tableaux of shape $\lambda$.

Proof: This is direct using the bijection between permutation tableaux and PT-words defined in Section 5. Indeed a PT-word corresponds to a L-Bell tableau (resp. R-Bell) if and only if each subword on the alphabet $\{U, V\}$ is of the form $U^{t} V^{n}$ where $t=1$ or 2 and $n \geq 0$ (resp. $U V^{n} U^{t}$ where $t=0$ or 1 and $n \geq 0$ ). Given a word $A=a_{1} \ldots a_{n}$, we define $\bar{A}$ to be the word $a_{n} \ldots a_{1}$. Then given a PT-word $w=U A_{1} D^{b_{1}} U A_{2} D^{b_{2}} \ldots$ we define $I(W)=U \overline{A_{1}} D^{b_{1}} U \overline{A_{2}} D^{b_{2}} \ldots$. The function $I$ is an involution on the set of PT-words. The previous remarks imply that $W$ is a PT-word that corresponds to a L-Bell tableau if and only of $I(w)$ is a PT-word that corresponds to a R-Bell tableau. The shapes of the tableaux are the same, as is immediately implied by the result of section 5.2. We could also define this involution directly on the tableaux, but it is less straightforward.

\section{Conclusion and open problems}

In this paper we give two bijections between permutation tableaux and permutations that send the columns of the tableaux to the descent of the permutation. We also relate the superfluous ones of the tableaux to the number of occurrences of the pattern $(31-2)$ of the permutation. We then use this approach to enumerate permutations with one occurrence of the pattern $(31-2)$. We finally introduce Bell tableaux that are in bijection with set partitions. It is well known that set partitions are in one-to-one correspondence with 
permutations with no occurrences of the pattern 32-1 [3]. It would be interesting to find the statistic on permutation tableaux that has the same distribution as the number of occurrences of 32-1.

\section{References}

[1] E. Babson and E. Steingrimsson, Generalized permutation patterns and a classification of the Mahonian statistics, Sem. Lothar. Combin., Vol. 44, Art. B44b, 2000, 18 pp.

[2] A. Burstein, Some properties of permutation tableaux, Annals of Comb., to appear, 2007.

[3] A. Claesson, Generalized Pattern Avoidance, European Journal of Combinatorics, Vol. 22, 2001, 961-971.

[4] A. Claesson and T. Mansour, Counting Occurrences of a Pattern of Type $(1,2)$ or $(2,1)$ in Permutations, Adv. in Appl. Math, Vol. 29, 2002, 293-310.

[5] S. Corteel, Crossings and alignments of permutations, Adv. in Appl. Math, Vol. 38, Issue 2, 2007, 149-163.

[6] S. Corteel and P. Nadeau, Bijections for Permutation Tableaux, European Journal of Combinatorics, to appear.

[7] S. Corteel, E. Steingrimsson and L. Williams, Permutation tableaux and Stirling numbers, in preparation, 2007.

[8] S. Corteel and L. Williams, Permutation tableaux and the asymmetric exclusion process, Adv. in Appl. Math, Vol. 39, Issue 3, 2007, 293-310.

[9] S. Corteel and L. Williams, A Markov chain on permutation tableaux which projects to the PASEP, Int Math Res Notices, Vol. 17, Art. ID rnm055, 2007.

[10] J. Françon and G. Viennot, Permutations selon leurs pics, creux, doubles montées et doubles descentes, nombres d'Euler et nombres de Genocchi, Discrete Mathematics, Vol. 28, Issue 1, 1979, 21-35.

[11] P. Nadeau, Chemins et Tableaux, Contributions à des problèmes de combinatoire énumérative et bijective, PhD thesis, Université Paris-Sud, 2007.

[12] A. Postnikov, Total positivity, Grassmannians, and networks. Preprint 2006. arXiv:math/0609764

[13] E. Steingrímsson and L. Williams, Permutation tableaux and permutation patterns, Journal of Combinatorial Theory, Series A, Vol. 114, Issue 2, 2007, 211-234.

[14] X. Viennot, Catalan tableaux, permutation tableaux and the asymmetric exclusion process, FPSAC07, Tianjin, China.

[15] L. Williams, Enumeration of totally positive Grassmann cells, Advances in Math, 190 (2005), 319342. 